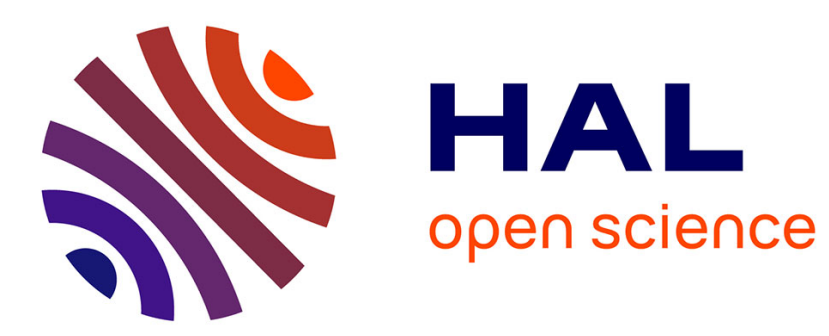

\title{
Theory of the memory effect in thiourea. Defect density waves in modulated systems
}

P. Lederer, G. Montambaux, J.P. Jamet, M. Chauvin

\section{To cite this version:}

P. Lederer, G. Montambaux, J.P. Jamet, M. Chauvin. Theory of the memory effect in thiourea. Defect density waves in modulated systems. Journal de Physique Lettres, 1984, 45 (12), pp.627-637. 10.1051/jphyslet:019840045012062700 . jpa-00232391

\section{HAL Id: jpa-00232391 https://hal.science/jpa-00232391}

Submitted on 1 Jan 1984

HAL is a multi-disciplinary open access archive for the deposit and dissemination of scientific research documents, whether they are published or not. The documents may come from teaching and research institutions in France or abroad, or from public or private research centers.
L'archive ouverte pluridisciplinaire HAL, est destinée au dépôt et à la diffusion de documents scientifiques de niveau recherche, publiés ou non, émanant des établissements d'enseignement et de recherche français ou étrangers, des laboratoires publics ou privés. 
Classification

Physics Abstracts

$61.70-64.70 \mathrm{~K}-66.30 \mathrm{~J}$

\title{
Theory of the memory effect in thiourea. Defect density waves in modulated systems
}

\author{
P. Lederer, G. Montambaux, J. P. Jamet and M. Chauvin \\ Laboratoire de Physique des Solides $\left({ }^{+}\right)$, Bât. 510, Centre d'Orsay, 91405 Orsay Cedex, France
}

(Reçu le 14 mars 1984, accepté le 19 avril 1984)

\begin{abstract}
Résumé. - Une approche Landau-Ginzburg simple rend compte qualitativement et semi-quantitativement des anomalies de susceptibilité et de biréfringence liées à l'effet mémoire. Le couplage gradient amplitude joue, dans la thiourée, un rôle déterminant. Nous démontrons la possibilité de créer, autour d'un point arbitraire dans la partie modulée du diagramme de phase, une phase incommensurable bloquée induite par défauts... Ceci arrive grâce à l'apparition d'une Onde de Densité de Défauts lorsque ceux-ci, mobiles, ont pu interagir pendant un temps suffisant avec une modulation statique. Nous discutons brièvement les conséquences pour la physique des systèmes modulés.
\end{abstract}

\begin{abstract}
A simple Landau-Ginzburg theory is shown to account qualitatively and semi-quantitatively for the memory effect anomalies of the susceptibility and the birefringence in thiourea. The importance of the gradient amplitude coupling energy in thiourea is emphasized. The possibility of creating a defect-induced locked incommensurate phase around any arbitrary temperature within the modulated phase is demonstrated. Such a locked phase will appear as a consequence of the condensation of a periodic defect concentration if extrinsic mobile impurities are allowed to interact for a sufficient time with a static modulation. We briefly discuss some aspects of such defect density waves in the physics of modulated structures.
\end{abstract}

A new memory effect, characteristic of modulated structures, was recently observed in thiourea [1]. Similar observations in various other systems were subsequently reported [2-4]. The memory was proved in reference [1] to be a periodicity memory : a thiourea crystal is kept during a few minutes at a constant arbitrary temperature $T^{*}$ and constant field $E^{*}$ in the modulated part of the phase diagram, i.e. a point where the modulation wave vector is $q^{*}=q\left(T^{*}, E^{*}\right)$; a susceptibility anomaly is observed when after cooling the system and heating again in an external field, the trajectory of the system in the phase diagram crosses the line $q(T, E)=q^{*}$.

The interpretation of the effect described in [1] was based on the idea that a concentration of defects such as impurities, vacancies, dislocation, etc., with non zero mobility acquires a periodic component if allowed to interact for a sufficient time with a static modulation. In the following we use the word impurities as a generic name for extrinsic defects (as opposed to intrinsic defects of the modulated order parameter, such as solitons, vortex lines, etc.).

New experimental results, obtained after a thiourea crystal was kept at a constant arbitrary

$\left(^{+}\right)$Associé au C.N.R.S. 
temperature $T^{*}$ and zero field (with $T^{*}$ in the modulated part of the phase diagram) during a much longer time (about 15 hours) than previously have been reported in part recently [5]. Figure 1a exhibits the observed effects on the susceptibility with increasing and decreasing $T$. Figure $1 \mathrm{~b}$ shows the birefringence anomaly (reported here for the first time). The susceptibility anomalies are very similar to the ones observed in the case of the commensurate phase [6] with $q=b^{*} / 8$ at fields higher than $500 \mathrm{~V} / \mathrm{mm}$. Accordingly, it was suggested in reference [5] that the wave vector was locked at the incommensurate value $q^{*}$ over a temperature interval $\Delta T \approx 1.5 \mathrm{~K}$. (In the case of Ref. [5] $T^{*}=209.5 \mathrm{~K}$; so that $q^{*} \cong b^{*} / 7.15$.) The reason for this locking is of course the extrinsic commensurability potential due to the periodic ordering of mobile defects during the « write-up » time.

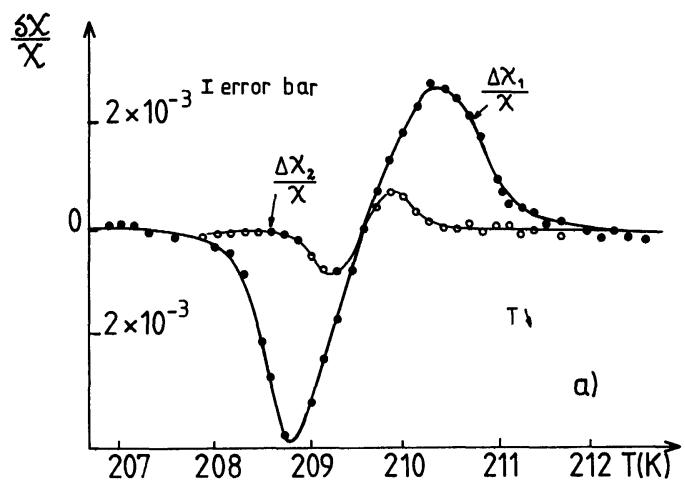

Fig. 1a. - Relative difference $\Delta \chi_{1} / \chi$ between the normal susceptibility and the one with the memory effect for decreasing $T$, after a 15 hours stabilization time at $T^{*}=209.6 \mathrm{~K}$ for decreasing $T ; \Delta \chi_{1} / \chi$ corresponds to the first experiment after the memory effect was "written-up ". The small amplitude curve $\Delta \chi_{2} / \chi$ corresponds to a second experiment after the system has been allowed to relax partially by heating it up to $3 \mathrm{~K}$ above $T_{\lambda}$ during 12 hours. From the ratio $\Delta \chi_{2} / \Delta \chi_{1}$ we deduce that the relaxation time of the defects is of the order of 12 hours at $T=T_{\lambda}+3 \mathrm{~K} \approx 220 \mathrm{~K}$.

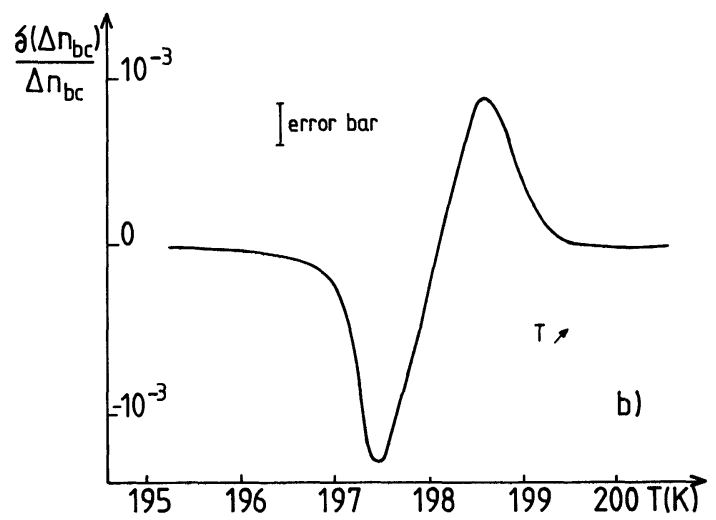

Fig. 1b. - Relative difference $\delta\left(\Delta n_{\mathrm{bc}}\right) / \Delta n_{\mathrm{bc}}$ between the normal birefringence and the one with the memory effect for increasing $T$, after a 95 hours stabilization time at $T=198.1 \mathrm{~K}$ for increasing $T$; this curve corresponds to the first experiment after the memory effect was written-up. The curve shown here is generic, and is similar to those obtained for different $T^{*}$. The fact that $T^{*}$ here corresponds to $q\left(T^{*}\right)=b^{*} / 8$ plays no role here : in zero field, there is no birefringence anomaly under a continuous temperature variation, and the modulation wave vector has no measurable anomaly, because there is no Umklapp term in zero field, as discussed in Refs. [6] and [11]. For details in experimental techniques, see Ref. [11]. 
In this Letter, we want to give a very simple theoretical description of the susceptibility anomaly (Fig. 1) which strongly supports the notion of a Defect-Induced Locked Incommensurate phase. We predict that a suitably conducted neutron diffraction investigation should give direct evidence for this lock in. Our theory is based on the consideration of the Landau-Ginzburg free energy appropriate to pure thiourea [6-9] and on the changes in the susceptibility due to a forced lock-in of the modulation wave vector.

\section{Free energy, susceptibility and birefringence.}

The standard Landau-Ginzburg functional appropriate to thiourea involves the component of the polarization along the ferroelectric axis $P_{x}(z)$ and its spatial derivatives along the anisotropy axis :

$$
F=\int\left\{\frac{1}{2} A_{0} P_{x}^{2}+\frac{1}{4} B P_{x}^{4}+\frac{1}{2} \alpha\left(\frac{\mathrm{d} P_{x}}{\mathrm{~d} z}\right)^{2}+\frac{1}{4} \gamma\left(\frac{\mathrm{d}^{2} P_{x}}{\mathrm{~d} z^{2}}\right)^{2}+\eta\left(P_{x} \frac{\mathrm{d} P_{x}}{\mathrm{~d} z}\right)^{2}-E P_{x}\right\} \mathrm{d} z
$$

$E$ is the applied electric field ; $A_{0}=\left(T-T_{0}\right) / C, B$ and $\gamma$ are positive constants and $\alpha$ is negative. As discussed in [7a] and [8], the term proportional to $\left(P_{x} \frac{\mathrm{d} P_{x}}{\mathrm{~d} z}\right)^{2}$ accounts for the curvature of the $q(T)$ curve below $T_{\lambda}$, the disordered modulated transition temperature. In fact the curvature of the curves $q(T, E)=$ const. is also well accounted for by this term. This is easily seen when the polarization wave is purely sinusoidal, that is, near enough the disordered-modulated transition line. We restrict our discussion to this case in the following. Then consider equation (1) with $P_{x}(z)=$ $P_{0}+2 P_{q} \cos q z$. It becomes [8]

$$
F=\frac{1}{2} A_{0} P_{0}^{2}+\frac{1}{4} B P_{0}^{4}+A_{q} P_{q}^{2}+\frac{3}{2} B_{q} P_{q}^{4}+\frac{3}{2}\left(B+B_{q}\right) P_{0}^{2} P_{q}^{2}-P_{0} E
$$

with

$$
A_{q}=A_{0}+\alpha q^{2}+\frac{1}{2} \gamma q^{4}
$$

and

$$
B_{q}=B+\frac{4}{3} \eta q^{2}
$$

Minimizing $F$ with respect to the amplitude $P_{q}$ in zero field, and then with respect to the modulation wave vector, one gets [8], when $T \rightarrow T_{\lambda}$ :

with

$$
q^{2}(T)-q^{2}\left(T_{\lambda}\right) \cong \mu\left(T-T_{\lambda}\right)
$$

$$
\mu=\frac{2}{3} \frac{\eta}{C \gamma B_{\lambda}}, \quad B_{\lambda}=B_{q}\left(T_{\lambda}\right)
$$

The curvature of the iso- $q$ lines in the $(E-T)$ plane is also easily obtained from (2) by minimizing with respect to $P_{0}$, to $P_{q}$ and then with respect to the modulation wave vector.

It is straightforward to find $P_{q}$ at all fields :

$$
P_{q}^{2}=-\frac{A_{q}}{3 B_{q}}-\frac{B+B_{q}}{2 B_{q}} P_{0}^{2}
$$

Birefringence is a direct measure of $P_{q}^{2}$, as discussed in reference [6]. 
The line $q(T, E) \equiv$ const. is given by :

$$
q^{2}=-\frac{\alpha}{\gamma}-\frac{2 \eta}{\gamma}\left(P_{q}^{2}+P_{0}^{2}\right)=\text { const. }
$$

whence, along the line $q=$ const.

$$
T(E)=T(E=0)+2 \eta q^{2} C P_{0}^{2} .
$$

Notice the relationship between the iso- $q$ line curvature, equation ( $\left.7^{\prime}\right)$, and the curvature of the curve $q(T, E=0)$ (Eq. (4)). Both are of course due to the gradient-amplitude coupling proportional to $\eta$; in zero-field, $q$ decreases when $P_{q}$ increases (because of the $q^{2} P_{q}^{4}$ term in (2)). In finite field, both terms $q^{2} P_{0}^{2} P_{q}^{2}$ and $q^{2} P_{q}^{4}$ contribute to the iso- $q$ line curvature.

As we shall see below, the amplitude of the memory effect susceptibility anomaly is directly related to those curvatures, quite an unexpected result at first sight. The pure system susceptibility at low field i.e. when $B P_{0}^{4} \ll A_{0} P_{0}^{2}$ and $P_{q}^{2} \gg P_{0}^{2}$ is immediately derived from (2) :

$$
\begin{aligned}
\chi_{0} & =1 /\left(A_{0}+3\left(B+B_{q}\right) P_{q}^{2}\right) \\
& =B_{q} /\left(A_{0} B_{q}-A_{q}\left(B+B_{q}\right)\right) .
\end{aligned}
$$

The unperturbed experimental susceptibility (i.e. in the absence of memory write-up) is shown on figure 2. Below about $210 \mathrm{~K}$, equation (8) is not adequate anymore and higher harmonics have to be taken into account, as discussed in reference [7a].

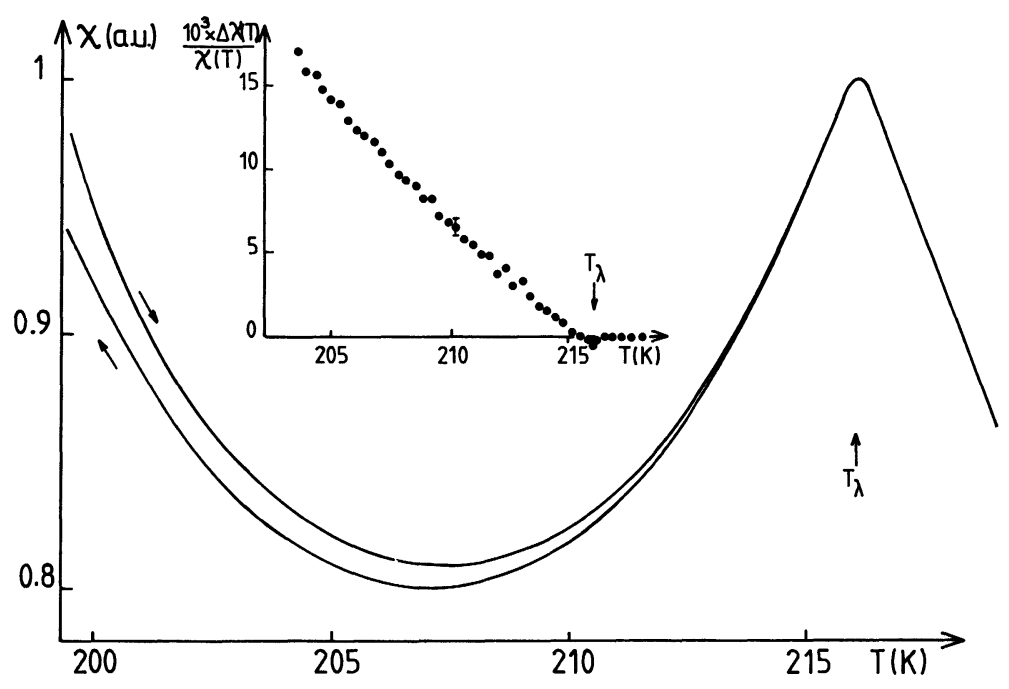

Fig. 2. - Unperturbed susceptibility curve for decreasing and increasing $T$. The curves have been arbitrarily normalized with respect to $\chi\left(T_{\lambda}\right)$ taken as unity. The insert shows the relative hysteresis $\Delta \chi(T) / \chi(T)$ as a function of $T . \Delta \chi(T) / \chi(T)$ is seen to go to zero at $T_{\lambda}$ as $\left(T-T_{\lambda}\right)$. This means, according to Eq. (11), that the modulation wave vector hysteresis should be finite at $T_{\lambda}$ (and only slowly varying in the whole modulated phase).

\section{The memory effect.}

We now assume that after the system has been stabilized during a sufficient time at a given tem- 
perature $T^{*}$, with modulation wave vector $q^{*}=q\left(T^{*}\right)$, a sinusoidal modulation of the impurity concentration appears, which generates a commensurability potential [1] $V_{\text {imp }}$

$$
V_{\mathrm{imp}}=V_{0} c_{0} b_{0}^{\prime}\left|P_{q}\right|^{2} \delta\left(q, q^{*}\right)
$$

where $c_{0}$ is the uniform impurity concentration and $b_{0}^{\prime}$ the fraction of impurities which have relaxed in the modulation periodic potential. (The impurity modulation interaction is assumed to be $V_{0} P^{2}(r)$.) The assumption of a sinusoidal impurity density is not a serious loss of generality and is made here for simplicity. In the simplest picture where impurities can be described by a single diffusion constant, it is easy to solve the diffusion equation in a periodic potential $V(r)=$ $V_{0} P^{2}(r)$ :

$$
\frac{\partial c}{\partial t}=D \Delta c+\frac{D}{k_{\mathrm{B}} T} \nabla(c \nabla V) \text { where } D \text { is the diffusion constant } .
$$

In this diffusion equation, $c$ is the concentration : $c(r)=c_{0}\left(1+b_{0}^{\prime} \sin 2 q z\right)$. One gets, in the limit $V_{0} P_{q}^{2} / k_{\mathrm{B}} T<1$

$$
b_{0}^{\prime}=\frac{V_{0} P_{q}^{2}}{k_{\mathrm{B}} T}\left[1-\exp \left(-4 D q^{*^{2}} t\right)\right]
$$

where $t$ is the « write-up " time. Thus, the relevant relaxation time for a population of defects with a single diffusion constant $D$ is $\tau=\left(4 D q^{2}\right)^{-1}$, i.e. impurities need not diffuse more than a fraction of a modulation wavelength to cause a memory effect.

Because of (9), we expect the modulation wave vector to be locked at $q^{*}$ within a temperature interval [6] :

with

$$
\Delta T=\frac{\sqrt{2}}{\pi} \frac{\left(V_{\mathrm{imp}} P_{q}^{-2}\right)^{1 / 2}}{|\alpha|^{1 / 2} \zeta}
$$

$$
\zeta=\left.\frac{\mathrm{d} q}{\mathrm{~d} T}\right|_{q=q^{*}} \simeq \frac{\mu}{2 q^{*}} .
$$

Putting $\left(9^{\prime}\right)$ into (10), we have

$$
\Delta T=\frac{\sqrt{2}}{\pi} \frac{c_{0}^{1 / 2} V_{0} P_{q}}{\left(k_{\mathrm{B}} T\right)^{1 / 2} \zeta|\alpha|^{1 / 2}} \quad \exp \left(-4 D q^{*^{2}} t\right)^{1 / 2}
$$

which varies as $t^{1 / 2}$ when $t \ll\left(4 D q^{*^{2}}\right)^{-1}$. These expressions are valid when $b_{0}^{\prime} \ll 1$ i.e. when $V_{0} P_{q}^{2} \ll k_{\mathrm{B}} T$. Derived for $\eta=0$, equation (10) is also valid for $\eta \neq 0$. In thiourea, equation (10') holds at $210 \mathrm{~K}$ for $V_{0}<2 \mathrm{eV}$; estimating $V_{0}=0.2 \mathrm{eV},\left(10^{\prime}\right)$ yields $c_{0} \simeq 3 \times 10^{-5}$ for $\Delta T \simeq$ $0.75 \mathrm{~K}$; if $V_{0}=0.02 \mathrm{eV}, c_{0} \simeq 3 \times 10^{-3}$. Given the strong dipolar coupling in the planes perpendicular to the anisotropy axis, (which are ferromagnetically ordered well above $T_{\lambda}$ ) such orders of magnitude are quite reasonable if the impurities are point defects.

On either side of the locked phase, we expect discommensurations [10] to allow a continuous relaxation of the wave vector to its unperturbed value.

The result is shown on figure 3 .

\section{The susceptibility anomaly and birefringence anomaly.}

In principle $V_{\text {imp }}$ also induces a change in $P_{q}$ at $T^{*}=T$ since one must add a term like (9) to equation (2) and minimize again with respect to $P_{q}$. This was the only effect considered in refe- 


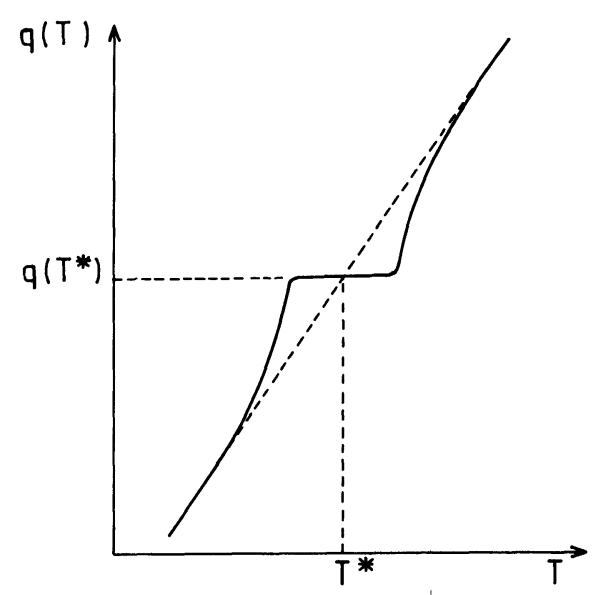

Fig. 3. - This figure may be viewed as the predicted outcome of a suitably conducted neutron diffraction measurement of the modulation wave vector, along the lines described in the caption of Fig. 1, or as the result of the susceptibility measurement of Fig. 1 suitably deduced from Eq. (11'), with $\xi=2.6$. We have assumed that the relaxation of $q(T)$ to its unperturbed value follows the law $q(T)-q\left(T^{*}\right)=\beta\left(T-T_{\mathrm{e}}\right)^{1 / 2}$ where $T_{\mathrm{e}}$ is the temperature of the edge of the locked step. The coefficient $\beta$ has been taken as determined experimentally in Ref. [1]. The edges of the step have been rounded off somewhat arbitrarily. The details of this behaviour do not matter for the purpose of this paper. One may use this figure for arbitrary $T^{*}$ and arbitrary $\Delta(T)$, provided $\Delta T \lesssim 3$ to $4 \mathrm{~K}$. In that case the unperturbed curve $q(T)$ is practically a straight line.

rence [1]. Here we assume this effect is negligible in comparison with the locking effect. This approximation is a good one, as will be discussed later on.

Thus, if we consider $\left(8^{\prime}\right)$ we find that the variation of $\chi$ due to the impurity induced modulation lock-in $\delta q(T)$ is

$$
\frac{\delta \chi(T)}{\chi(T)}=+\left(\frac{4}{3} \frac{\eta q^{2}}{B_{q}}\right)^{2} A_{q} \chi \frac{\delta q(T)}{q} .
$$

Since $A_{q}=\left(T-T_{\lambda}\right) / C$ where $T_{\lambda}$ is the paraelectric-modulated transition line, the sign of $\delta \chi / \chi$ is opposite to that of $\delta q / q$. Thus $\delta \pi(T)$ is a slightly deformed snapshot of $-\delta q / q$. The smaller $\Delta T$, the lesser the deformation.

Defining $\xi=\frac{4}{3} \eta q^{2} / B,(11)$ can be rewritten

$$
\frac{\delta \chi}{\chi}=-\left(\frac{\xi}{\xi+1}\right)^{2}\left(\frac{T-T_{0}}{T_{\lambda}-T}+\frac{\xi+2}{\xi+1}\right)^{-1} \frac{\delta q}{q}
$$

( $\xi$ is weakly temperature dependent because it is proportional to $q^{2}(T)$ ). $\delta q(T)$ is derived from figure 3. The result is plotted in figure $4 \mathrm{a}$ where it is compared to the experimental result. There is no adjustable parameter since $\xi \cong 2.6$, as explained below and $2 \Delta T$ in figure 3 has been assumed to be $1.5 \mathrm{~K}$.

Birefringence measurements [6] measure $P_{q}^{2}$. Forcing the modulation wave vector to stay locked around $T^{*}$ induces a change $\delta P_{q}^{2}$ due to the $\eta q^{2} P_{q}^{4}$ term in equation (2). We have straightforwardly from (6)

$\delta P_{q}^{2}=-\left\{P_{q}^{2}(E=0)+\frac{P_{0}^{2}}{\xi+1}\left[1+\frac{\xi}{\left(\frac{\left(T-T_{0}\right)}{\left(T_{\lambda}-T\right)} \frac{(\xi+1)}{(2+\xi)}+1\right)}\right]\right\} \frac{\xi \delta q(T)}{(\xi+1) q(T)}$. 
In zero field

$$
\delta P_{q}^{2} / P_{q}^{2}=-\frac{\xi}{(\xi+1)} \frac{\delta q(T)}{q}
$$

Birefringence measurements yield an undistorted snapshot of the modulation wave vector perturbation in zero field (Fig. 1b). Furthermore $\frac{\delta \chi}{\chi}$ is proportional to $\frac{\delta P_{q}^{2}}{P_{q}^{2}}$.

This is in obvious agreement with observation, as shown in figure $4 \mathrm{~b}$. The latter exhibits another experimental check of the theory, namely the absence of detectable asymmetry in the birefringence anomaly, in contradiction with the asymmetry of the susceptibility one. There is again no adjustable parameter in figure $4 \mathrm{~b}$, since $\xi \cong 2$ at $198 \mathrm{~K}$ is determined from $\xi=2.6$ at $210 \mathrm{~K}$, as explained below.

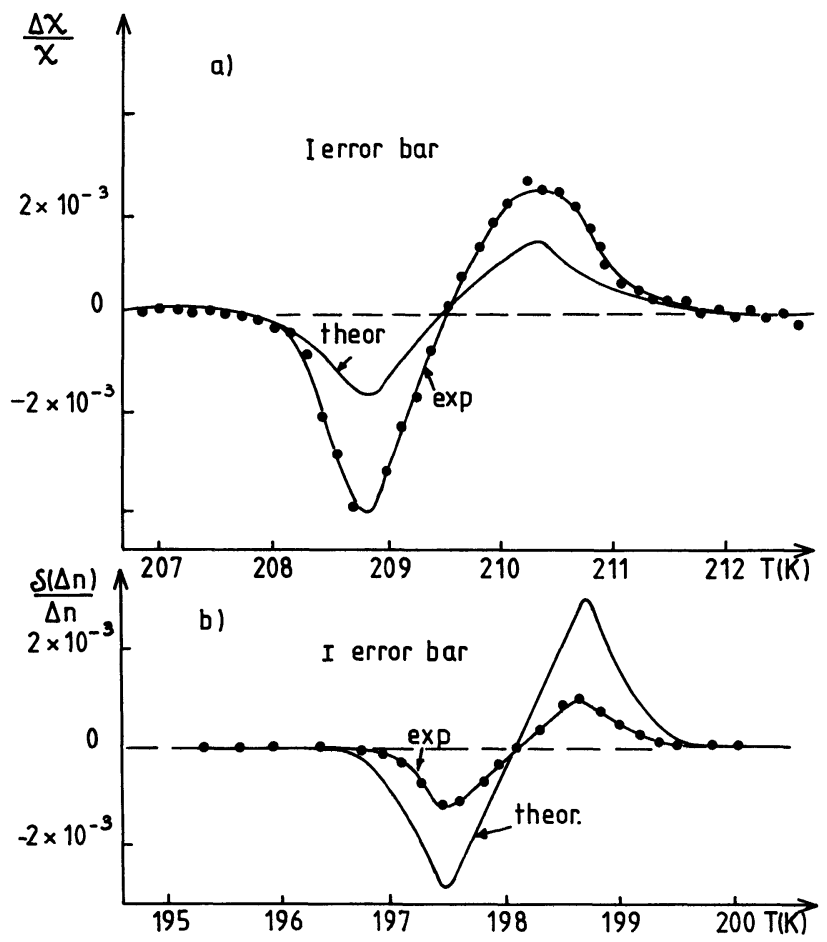

Fig. 4. - Comparison between experimental data and theory. Fig. 4a compares the data for the susceptibility anomaly with Eq. $\left(11^{\prime}\right)$ where $\delta q / q$ is deduced from Fig. 3. Fig. 4 b deals with the birefringence anomaly and Eq. $\left(12^{\prime}\right)$. In Fig. $4 \mathrm{a}, \xi=2.6$, as explained in the text; $T=209.6 \mathrm{~K}$ and $2 \Delta T \simeq 1.5 \mathrm{~K}$. In Fig. $4 \mathrm{~b}$, $\xi$ has to be corrected for the variation of $q^{2}(T)$, because $T^{*}=198.5 \mathrm{~K}$, so that $\xi \simeq 2$ and $2 \Delta T \simeq 1 \mathrm{~K}$.

As mentioned earlier in this letter, equations (5), ( $\left.7^{\prime}\right),(11)$ and (12) establish relationships between the iso- $q$ lines curvature, the $q(T)$ curve curvature, susceptibility and birefringence memory anomalies. Other features of the thiourea phase diagram $[6,7 \mathrm{~b}]$ help establish coefficients and evaluate numerically the amplitude of the anomalies in $\chi$ and birefringence. The only assumption needed to do it is that $\delta q(T)$ is described by figure 2, as a result of impurity induced self commensurability potential. 
It is known that $T_{\lambda}=216 \mathrm{~K}, T_{0} \simeq 203 \mathrm{~K} \pm 3 \mathrm{~K}$. From $\left(7^{\prime}\right)$ one has, using the data in reference [1]

$$
\begin{aligned}
C \eta q^{2} & =\frac{1}{2} \frac{T(E)-T(E=0)}{\chi_{0}^{2} E^{2}} \\
& \simeq \frac{1}{2}\left(T-T_{0}+\left(T_{\lambda}-T\right)\left(\frac{2+\xi}{\xi+1}\right)\right)^{2} \text { at } T=210 \mathrm{~K} .
\end{aligned}
$$

A reasonable choice for $\xi$ is $\xi \sim 1$; this yields (for $\xi=1$ ), $C \eta q^{2}=128 \mathrm{~K}$ so that $B \simeq 171 \mathrm{~K}$. Since $B$ must be of order $T_{0}=203 \mathrm{~K}$, the choice $\xi \approx 1$ is seen to be quite good. From $T_{\lambda}-T_{0}=$ $\frac{C}{2} q^{4} \gamma$ we deduce $C \gamma q^{4}=26 \mathrm{~K}$ (at $T_{\lambda}$ ) so that from equation (5) we get $\xi \simeq 2.6$; this value is also quite close to the estimate which can be made from the value of $T_{\lambda}, T_{0}$, and $T_{\mathrm{H}}$, the first order transition temperature to the ferroelectric phase. The only quantity needed in $\left(11^{\prime}\right)$ is $\xi$.

Taking $\xi=2.6$ one has

$$
\frac{\delta \chi}{\chi} \simeq-0.2 \frac{\delta q}{q} \text { and } \delta P_{q}^{2} / P_{q}^{2} \simeq-0.7 \frac{\delta q}{q}
$$

The theoretical figure for the maxima of $\frac{\delta q}{q}$ is (from Fig. 3)

$$
\frac{\delta q}{q}=\frac{1}{q} \frac{\mathrm{d} q}{\mathrm{~d} T} \Delta T \sim 7 \times 10^{-3} \text { for } \Delta T=0.75 \mathrm{~K} .
$$

Experimentally, $\left(\frac{\delta \chi}{\chi}\right)_{\max } \simeq 2 \times 10^{-3}$, and $\left(\delta P_{q}^{2} / P_{q}^{2}\right)_{\max } \simeq 10^{-3} \pm 0.2 \times 10^{-3}$. The experimental result for the birefringence is about $30 \%$ of the theoretical figure, again with no adjustable parameter. $T^{*}$ in figure $4 \mathrm{~b}$ is outside the region of validity of the single harmonic approximation. (At higher temperatures, the amplitude of the anomaly decreases like $P_{q}^{2}$.) It is possible that the agreement between theory and experiment about the birefringence anomaly improves when higher order harmonics are taken into account. We consider anyway that an order of magnitude agreement is a proof of the validity of our analysis.

Figures $4 \mathrm{a}$ and $4 \mathrm{~b}$ exhibit various satisfactory features :

- the overall shape of $\delta \chi / \chi$ and $\delta(\Delta n) / \Delta n$ is qualitatively well accounted for ;

- in particular the negative part is always at $T<T^{*}$, the positive part at $T>T^{*}$, as observed. As evidenced from figure 3 this is a straightforward consequence of the positive derivative of the $q(T)$ curve ;

- the zero of the anomaly is at $T=T^{*}$, as observed experimentally within experimental accuracy. This shows that at exactly $T^{*}$ the variation of $P_{q}^{2}$ due to $V_{\text {imp }}$ is negligible, as was assumed above. Thus thiourea is shown to be a system where the impurity modulation interaction can be treated as weak. Obviously there exist in nature systems where this is not so. In that case the amplitude of $P_{q}$ at exactly $T=T^{*}$ increases with the write up time and saturates if the latter is longer than the relaxation time. We believe that such is the case in BSN [4] (baryum sodium niobate);

- the low $T$-high $T$ asymmetry is qualitatively well accounted for, and also semi-quantitatively. The main reason for it is the temperature variation of $A_{q}$ (proportional to $P_{q}^{2}$ ) in (11).

From (11') using $T_{0}=203 \mathrm{~K}$ and $\xi=2.6$, we find for the ratio of the two maxima of $\delta \chi$ below and above $T^{*}: \delta \chi_{+} / \delta \chi_{-}=1.25$ as compared with about $1.60 \pm 0.20$ experimentally. Critical fluctuations improve the agreement since $A_{q} \sim\left(T_{\lambda}-T\right)^{0.75}$ varies more rapidly than $\left(T_{\lambda}-T\right)$. 
- There is no singularity in the experimental $\delta \chi(T)$ curve where the turning points are rounded off.

This last point is not surprising. Static impurities are known to round off the commensurate incommensurate transition. In fact we expect neutron diffraction experiment, if sufficiently accurate, to show that edges are indeed rounded off as $\delta \chi(T)$ shows.

\section{Global hysteresis of the modulated phase.}

The modulated phase of thiourea exhibits global hysteresis of the modulation wave vector [16] as well as of the susceptibility, birefringence, locked phase under field, etc... [16] (see Fig. 2). In fact neutron diffraction curves are usually measured point by point with a time of about one hour per point. This is too short a time to produce a sizeable wave vector locking. In other words measurements done until now represent the property of the system when defects, impurities, etc... are at random, practically frozen during the experiment (what we would call a « fast experiment "). The extrinsic nature of this hysteresis is suggested by the large amplitude memory effect shown in figure $1 \mathrm{a}$ and $1 \mathrm{~b}$ (of the order of the global hysteresis). This invalidates speculations that global hysteresis is a consequence of devil's stair case effects [17]. Another proof of this is given by noting that equation (11) describes semi-quantitatively (without any adjustable parameter, since we know $\xi \sim 2$ ) the relationship between global hysteresis on the modulation wave vector and that on the susceptibility.

Experimentally $\frac{\delta q}{q} \simeq 2 \times 10^{-2} \pm 10^{-2}$ and $\delta \chi / \chi \simeq 8 \times 10^{-3} \pm 10^{-3}$ at $208 \mathrm{~K}$; the theoretical estimate is, with $\xi=2.6, \delta \chi / \chi=4 \times 10^{-3} \pm 2 \times 10^{-3}$. Similarly $\frac{\partial P_{q}^{2}}{P_{q}^{2}}=-\frac{2}{3} \frac{\partial q}{q}$, roughly as observed experimentally.

The following argument suggests that the modulation wave vector global hysteresis should be roughly constant up to $T_{\lambda}$. Indeed the thermodynamic restoring force should balance the average force due to the modulation interaction with all defects (mobile or frozen). The latter should be $\sim W^{\prime}(z) P_{q}^{2}$ where $W^{\prime}(z)$ is an effective average gradient describing the intensity of this force. Thus

$$
8 \alpha q^{2}(\delta q(T) / q) P_{q}^{2} \simeq q^{-1} W^{\prime} P_{q}^{2}
$$

so that $\delta q(T) / q= \pm W^{\prime} / 8 \alpha q^{3}$, roughly independent of temperature. Equation $\left(11^{\prime}\right)$ also predicts $\delta q(T) / q$ independent of temperature, since, experimentally $\delta \chi / \chi$ goes to zero at $T_{\lambda}$ like $T-T_{\lambda}$. Experimental data is needed to check this point. Note that we estimate $q^{-1} W^{\prime} \sim W \simeq 8 \alpha q^{2} \times$ $(\delta q / q) \simeq 2 \mathrm{~K}$ per atom

\section{Discussion and conclusion.}

We believe that we have given a convincing analysis of the large amplitude memory effect described in part in reference [5] and shown in figures 1a and $1 \mathrm{~b}$ as an Impurity Induced Locked Incommensurate Phase as suggested in [5]. The ultimate proof would be a direct neutron diffraction experiment on a crystal held for a sufficient time at an arbitrary temperature within the modulated phase. The experiment is under way at the moment $[15 b]$; (after all, the existence of a locked phase of commensurability 8 under field was predicted on the basis of a theoretical analysis of susceptibility anomalies [11] and checked by neutron diffraction a few months later [12]). Various recent results increase our confidence. On one hand, X-ray studies of thiourea at high fields $(E>1200 \mathrm{~V} / \mathrm{mm})$ have been claimed to exhibit intrinsic lock-in [13] at high commensurability orders $15,29,43$, contrary to all known results obtained from susceptibility [6], birefringence [6] or neutron diffraction studies [14], and contrary to theoretical expectations based on a reasonably 
successful Landau-Ginzburg theory of the phase diagram [6]. Devil's staircase effects (lattice discreteness effects) are claimed in reference [13] to be found on the basis of an incorrect extension at high fields of a formula for discommensuration widths valid only at low fields [13, 6]. On the other hand, it has been shown recently that X-ray irradiation creates defects in a thiourea crystal, and changes thoroughly the thermal neutron diffraction pattern of the modulated phase under field [14] as well as the dielectric properties [15a]. Such defect generation is absent from a thermal neutron diffraction experiment in thiourea.

Our own memory effect observations and analysis complement the above results and support the notion that the lock-in reported in reference [13] are extrinsic effects due to irradiation defects $[14,15,18]$. Whether irradiation defects are frozen or mobile remains to be established by further studies.

The notion of a periodic modulation of concentration of defects is at variance with practically all the standard theories of impurities in modulated systems, which assume a frozen concentration of random defects $[19,20]$. Such theories are thus seen to be relevant to " fast experiments ", i.e. experiments such that impurity diffusion processes are negligible. In the physics of modulated structures, it appears crucial to establish, whether one deals with " fast experiment properties » or with " slow" ones, where memory effects will play a role. Thiourea, as well as many other modulated systems, has the property that due to the gradient amplitude coupling, memory effects are large and easily detected by simple measurements. But many modulated systems with weaker or no such coupling will not exhibit the same memory effect on birefringence, and a much smaller one on susceptibility, although a periodic modulation of defect concentration must be present. For instance, in most metallic systems where charge density waves and periodic lattice distortions occur, the modulation wave vector varies little or not at all with temperature because it is pinned at twice the Fermi wave vector $k_{\mathrm{F}}$ (quasi 1-D systems). In such systems, most experiments are likely to be " slow " ones, because the modulation is static. Then impurities will contribute a potential of periodicity $2 k_{\mathrm{F}}$ which modifies the electronic gap. The threshold field for non ohmic conductivity depends on the amplitude of the Defect Density Wave and one expects a periodic modulation of the current above threshold, as already mentioned about the memory effect [1]. Memory effects on the conductivity have actually been observed and reported for a typical CDW system like $\mathrm{TaS}_{3}[21]$ and for the blue bronzes [22]. Whether they can be accounted for qualitatively and quantitatively by a periodic defect concentration remains to be seen. However the possibility is a very serious one. The occurrence of Defect Density Waves is a serious possibility in all modulated systems. In all modulated structures, metallic or insulating, the Defect Density Wave introduces a gap in the phason spectrum, the amplitude of which is given by equation (9) or its equivalent in the case of a linear coupling between impurities and order parameter (Charge Density Wave case). Defect Density Waves are expected to have a generally smaller amplitude in Spin Density Wave Systems because of the weaker spin density coupling to the lattice. Although new in the field of modulated structures, the notion of a Defect Density Wave seems a very natural one when looked at from the physics of dislocations in solids, from that of ferroelectricity or from that of magnetic materials. It is well known that impurities decorate dislocations [23] (PortevinLe Chatelier effect), ferromagnetic or ferroelectric domain walls [24]. The novel aspect of this in the case of modulated structures is the periodicity of the concentration modulation.

Let us point out that our detailed understanding of the memory effect through the gradientamplitude coupling term allows to improve our understanding of the pure system phase diagram. Indeed, the simple theory described in reference [6] ignores the $\eta$ term, although it.was pointed out there that it should be taken into account in an improved version. Obviously this is especially true for the quantitative analysis of the birefringence anomalies (see Fig. 1 in Ref. [6]) and of the susceptibility ones which are better understood along the lines described here : in that case we have a few available neutron diffraction curves exhibiting the locked phase of order $8[12,14,15]$. We can use those to reconstruct the " unperturbed " birefringence and susceptibility curves i.e. 
in the absence of Umklapp term. This allows also to estimate the magnitude of higher modulation harmonics effects [25].

An improved quantitative agreement between theory and experiment is expected if one takes into account higher order harmonics along the lines of reference [7a].

There remains to identify the defects which participate in memory effects. It seems this has been done in at least one instance : sodium vacancies are believed to be the responsible agents in BSN $[3,4]$. In thiourea, this question is still unanswered. Mastering the chemistry of impurities in modulated structures is a key to interesting developments both in fundamental physics and in technology.

\section{References}

[1] JAMEt, J. P. and Lederer, P., J. Physique Lett. 44 (1983) L-257.

LEDERER, P. and JAMET, J. P., Proceedings of the SFP conference (Grenoble 83).

[2] Unruh, H. G., J. Phys. C 16 (1983) 3245.

[3] Errandonea, G., Toledano, J. C., Litzler, A., Savary, H., Schneck, J. and Aubrée, J., preprint (1983).

[4] Schneck, J., Calvarin, G. and Kiat, J. M., Phys. Rev. B 29 (1984) 1476.

[5] JAMET, J. P., Lederer, P., Ferroelectrics Lett. 1 (1984) 139.

[6] Barreto, M., Lederer, P. and JAmet, J. P., Phys. Rev. B 28 (1983) 3994.

[7] a - IsHiBASHI, Y. and SHIBA, H., J. Phys. Soc. Jpn 45 (1978) 409.

b - Lederer, P. and Chaves, C. M., J. Physique Lett. 42 (1981) L-127.

[8] Durand, D., Denoyer, F., Lefur, D., Currat, R. and Bernard, L., J. Physique Lett. 44 (1983) L-207.

[9] See also Hornreich, R. M., Luban, M. and Shtrickman, S., Phys. Rev. Lett. 35 (1975) 1678.

Michelson, A., Phys. Rev. B 16 (1977) 577.

Coutinho Filho, M. E. and De Moura, M. A., J. Magn. Magn. Mater. 15-18 (1980) 433.

[10] McMillan, W., Phys. Rev. B 14 (1976) 1496.

DzyaloshinskiI, I. E., Sov. Phys. JETP 20 (1965) 665.

[11] Jamet, J. P., Lederer, P., Moudden, A. H., Phys. Rev. Lett. 48 (1982) 442.

[12] Moudden, A. H., Svensson, E. C. and Shirane, G., Phys. Rev. Lett. 49 (1982) 557.

[13] Moudden, A. H., Moncton, D. E. and Axe, J. D., Phys. Rev. Lett. 51 (1983) 2390.

[14] Durand, D., Denoyer, F., Currat, R. and Vettier, C., to be published.

[15] a - André, G., Durand, D., Denoyer, F., to be published.

b - ANDRÉ, G., DuRAND, D., DenOYER, F., private communication.

[16] Moudden, A. H., Thèse d'état, Orsay (1980).

[17] Aubry, S., J. Physique 44 (1983) 147.

[18] André, G., Currat, R., Denoyer, F., Durand, D., Jamet, J. P., Lederer, P., Montambaux, G., to be published.

[19] McMillan, W. L., Phys. Rev. B 12 (1975) 1187.

[20] LeE, P. A. and Rice, T. M., Phys. Rev. B 19 (1979) 3970.

[21] Mihaly, G. and Mihaly, L., Solid. State Commun. 48 (1983) 449.

[22] Dumas, J. and Schlenker, C., to be published in Proceedings of Int. Symp. on Non-Linear Transport and Related Phenomena in Inorganic Quasi 1-D Conductors, Sapporo, Japan (1983).

[23] Friedel, J., Dislocations, chap. XIII (Pergamon Press) 1964.

[24] LAJZEROWICZ, J., private communication.

[25] Lederer, P. and Montambaux, G., to be published. 